\title{
Gamma-ray emission expected from Kepler's supernova remnant
}

\author{
E. G. Berezhko ${ }^{1}$, L. T. Ksenofontov ${ }^{1}$, and H. J. Völk ${ }^{2}$
}

1 Yu. G. Shafer Institute of Cosmophysical Research and Aeronomy, 31 Lenin Ave., 677980 Yakutsk, Russia e-mail: berezhko@ikfia.ysn.ru

2 Max-Planck-Institut für Kernphysik, Postfach 103980, 69029 Heidelberg, Germany

e-mail: Heinrich.Voelk@mpi-hd.mpg.de

Received 3 January 2006 / Accepted 19 January 2006

\section{ABSTRACT}

\begin{abstract}
Aims. Nonlinear kinetic theory of cosmic ray (CR) acceleration in supernova remnants (SNRs) is used to investigate the properties of Kepler's SNR and, in particular, to predict the $\gamma$-ray spectrum expected from this SNR.

Methods. Observations of the nonthermal radio and X-ray emission spectra as well as theoretical constraints for the total supernova $(\mathrm{SN})$ explosion energy $E_{\mathrm{sn}}$ are used to constrain the astronomical and particle acceleration parameters of the system.

Results. Under the assumption that Kepler's SN is a type Ia SN we determine for any given explosion energy $E_{\mathrm{sn}}$ and source distance $d$ the mass density of the ambient interstellar medium (ISM) from a fit to the observed SNR size and expansion speed. This makes it possible to make predictions for the expected $\gamma$-ray flux. Exploring the expected distance range we find that for a typical explosion energy $E_{\mathrm{sn}}=10^{51} \mathrm{erg}$ the expected energy flux of TeV $\gamma$-rays varies from $2 \times 10^{-11}$ to $10^{-13} \mathrm{erg} /\left(\mathrm{cm}^{2} \mathrm{~s}\right)$ when the distance changes from $d=3.4 \mathrm{kpc}$ to $7 \mathrm{kpc}$. In all cases the $\gamma$-ray emission is dominated by $\pi^{0}$-decay $\gamma$-rays due to nuclear CRs. Therefore Kepler's SNR represents a very promising target for instruments like HESS, CANGAROO and GLAST. A non-detection of $\gamma$-rays would mean that the actual source distance is larger than $7 \mathrm{kpc}$.
\end{abstract}

Key words. cosmic rays - acceleration of particles - ISM: supernova remnants - radiation mechanisms: non-thermal gamma rays: theory - hydrodynamics

\section{Introduction}

Kepler's supernova remnant (SNR) $(\mathrm{G} 4.5+6.8)$ is the result of a bright supernova (SN) in our Galaxy that exploded in 1604. This SNR has been extensively observed throughout the electromagnetic spectrum (for a recent review, see Blair 2005, and references therein). At the same time the type of Kepler's SN has been debated over the years. Initially it was considered a type Ia SN, based on a study of the historical light curve of the SN (Baade 1943). In addition its location well above the Galactic plane would be unexpected for a massive progenitor star. More recently it was argued that the light curve does not contradict a type II-L SN (Doggett \& Branch 1985), and Bandiera (1987) proposed a bow-shock model in which a massive star, ejected from the Galactic plane, exploded into its own circumstellar medium. According to this picture the remnant now interacts with the dense bow-shock shell that was produced in the interaction of the progenitor's stellar wind with the interstellar medium. Subsequently Borkowski et al. (1992) calculated a detailed model of the SNR dynamics. It was argued that the observed morphology is in good agreement with the bow-shock model. However, the thermal X-ray spectra, obtained more recently with ASCA (Kinugasa \& Tsunemi 1999), Chandra (Hwang et al. 2000) and XMM (Cassam-Chenaï et al. 2004), and corresponding theoretical modeling (Badenes et al. 2005), favor a type Ia event. We take this as our starting point.

The similarity of their light curves and spectra favor the idea that $\mathrm{SNe}$ of type Ia form a rather homogeneous class with small variations in absolute brightness. This leads to the assumption that the corresponding mechanical explosion energy $E_{\mathrm{sn}}$ has also a very narrow distribution. Despite the considerable progress in modeling of type Ia SN explosions, up to now there is no consensus about the mean value of the explosion energy. Within the so-called delayed-detonation model a typical range $E_{\mathrm{sn}}=$ $(1.3-1.6) \times 10^{51} \mathrm{erg}$ was obtained (Gamezo et al. 2005). This agrees with the range obtained from several objects (Wheeler et al. 1995).

The deflagration model has resulted in considerably lower mean energy releases $E_{\mathrm{sn}}=(0.4-0.6) \times 10^{51} \mathrm{erg}($ Khokhlov 2000; Reinecke et al. 2002). The latter calculations however appear to underestimate to some extent the value of the explosion energy (Reinecke et al. 2002; Hillebrandt 2004), which is now expected to be rather in the range $E_{\mathrm{sn}}=(0.8-1) \times 10^{51} \mathrm{erg}$ in this approach (Hillebrandt, private communication). In this situation we use below the value $E_{\mathrm{sn}}=10^{51} \mathrm{erg}$ as a typical explosion energy for type Ia events. Since the value of $E_{\mathrm{sn}}$ strongly influences the SNR dynamics and in particular the expected $\gamma$-ray flux, we explore the range $E_{\mathrm{sn}}=(0.5-2) \times 10^{51} \mathrm{erg}$, in order to demonstrate the sensitivity of the final results to the value of $E_{\mathrm{sn}}$.

The most recent radio study of the distance to the SNR by Reynoso \& Goss (1999) leads to a lower limit of $4.8 \pm 1.4 \mathrm{kpc}$ and an upper limit of $6.4 \mathrm{kpc}$. Therefore we explore below the range $d=3.4-7 \mathrm{kpc}$. For any given pair of values $E_{\mathrm{sn}}$ and $d$ we find the density of the ambient interstellar medium (ISM) from a fit to the observed SNR size and expansion speed (Dickel et al. 1988). This makes it possible to make quite definite predictions for the cosmic ray (CR) and $\gamma$-ray production in this SNR. We apply here the nonlinear kinetic theory of $\mathrm{CR}$ acceleration in SNRs (Berezhko et al. 1996; Berezhko \& Völk 1997). As was successfully done for the remnants SN 1006, Cas A and Tycho's SNR (Berezhko et al. 2002, 2003a,b; Völk et al. 2002, 2005; 
Berezhko \& Völk 2004), we use observations of the nonthermal radio and X-ray emission spectra to constrain the astronomical parameters as well as the particle acceleration parameters of the system, such as the interior magnetic field strength and the $\mathrm{CR}$ injection rates. We show that in all the cases considered the expected $\gamma$-ray flux is at a detectable level if the source distance is not larger than $7 \mathrm{kpc}$. Therefore the detection of $\mathrm{TeV} \gamma$-rays from Kepler's SNR (that is expected in the near future) will enable us to determine the SN explosion energy and the source distance.

\section{Model}

Hydrodynamically, a SN explosion ejects a shell of matter with total energy $E_{\mathrm{sn}}$ and mass $M_{\mathrm{ej}}$. During an initial period the shell material has a broad distribution in velocity $v$. The fastest part of this ejecta distribution is described by a power law $\mathrm{d} M_{\mathrm{ej}} / \mathrm{d} v \propto v^{2-k}$, following the treatments by Jones et al. (1981) and Chevalier (1982). The interaction of the ejecta with the ISM - assumed to be uniform - creates a strong shock which heats the thermal gas and accelerates particles diffusively to a nonthermal CR component of comparable energy density. For SN Ia explosions we use the values $M_{\mathrm{ej}}=1.4 M_{\odot}$ and $k=7$. Note that since Kepler's SNR is already in the Sedov evolutionary phase its dynamics are not very sensitive to the ejecta parameters $M_{\mathrm{ej}}$ and $k$.

Our nonlinear kinetic theory for this process is based on a fully time-dependent, spherically symmetric solution of the $\mathrm{CR}$ transport equations, coupled nonlinearly to the gas dynamic equations for the thermal gas component. Since all relevant equations, initial and boundary conditions for this model have already been described in detail in the above papers, we do not present them here and only briefly discuss the most important aspects below.

The coupling between the ionized thermal gas (plasma) and the energetic particles occurs primarily through magnetic field fluctuations carried by the plasma which scatter energetic particles in pitch angle. In shock waves this allows the diffusive acceleration of particles. The plasma physics of the field fluctuations is not yet worked out in full detail. Nevertheless, the accelerating CRs strongly excite magnetic fluctuations upstream of the outer SN shock in the form of Alfvén waves (e.g. Bell 1978; Blandford \& Ostriker 1978) as a result of the so-called streaming instability. In quasilinear approximation the wave amplitudes $\delta B$ grow to very high values $\delta B>B$, where $B$ is the average field strength (McKenzie \& Völk 1982). Since such fluctuations scatter CRs extremely effectively, the CR diffusion coefficient can therefore be assumed to be as small as its asymptotic limit $\kappa(p)=\kappa(m c)(p / m c)$, the Bohm limit, where $\kappa(m c)=m c^{3} /(3 e B)$, $e$ and $m$ are the particle charge and mass, $p$ denotes the particle momentum, $B$ is the magnetic field strength, and $c$ is the speed of light.

If $B_{\text {ISM }}$ is the pre-existing field in the surrounding medium, then the strong streaming instability would suggest that the instability growth is restricted by nonlinear mechanisms to a level $\delta B \sim B_{\mathrm{ISM}}$, any further turbulent energy being dissipated into the thermal gas (Völk \& McKenzie 1981). Earlier attempts to give a full nonlinear description of the magnetic field evolution through a numerical simulation (Lucek \& Bell 2000; Bell \& Lucek 2001) concluded that a considerable amplification of the "average" magnetic field in the smooth shock precursor - produced by the finite CR pressure - should occur. It was expected that a non-negligible fraction of the shock ram pressure $\rho_{0} V_{\mathrm{s}}^{2}$ ( $\rho_{0}$ is the ISM density) is converted into magnetic field energy.
Subsequently, Bell (2004) argued that this amplification is the result of a nonresonant instability, giving rise to the effective upstream field $B_{0}>B_{\mathrm{ISM}}$, on top of which the Alfvén waves grow to amplitudes $\delta B \sim B_{0}$. The Bohm limit and the gas heating are then to be calculated with $B=B_{0}$.

In our analyses of the synchrotron spectrum of SN 1006, Tycho's SNR, and Cas A, such magnetic field amplifications were indeed found; they can only be produced as a nonlinear effect by a very effectively accelerated nuclear CR component. Its energy density, consistent with all existing data, is so high that it is able to strongly excite magnetohydrodynamic fluctuations, and thus to amplify the upstream magnetic field $B_{\text {ISM }}$ to an effective field $B_{0}>B_{\mathrm{ISM}}$, at the same time permitting efficient $\mathrm{CR}$ scattering on all scales, reaching the Bohm limit. The same large effective magnetic field turns out to be required, within the errors, by the comparison of this self-consistent theory with the field strength derived from the morphology of the observed $\mathrm{X}$-ray synchrotron emission, in particular its spatial fine structure. We therefore also allow for the possibility of an amplified field and assume the corresponding Bohm limit for the particle diffusion coefficient.

We describe the number of suprathermal protons injected into the acceleration process by a dimensionless injection parameter $\eta \ll 1$ which is a fixed fraction of the gas particles entering the shock front. For simplicity we assume that the injected particles have a velocity four times higher than the postshock sound speed (see Malkov \& Drury 2001, for a review). It is expected that ion injection is quite efficient at the quasiparallel portion of the shock surface, where it is characterized by values $\eta=10^{-4}$ to $10^{-3}$ (see Völk et al. 2003, for details). Since this injection is expected to be strongly suppressed at the quasiperpendicular parts of the shock, one should renormalize the results for the nucleonic spectrum which were calculated within the spherically symmetric model. The deviation from spherical symmetry, introduced by the divergence-free nature of the magnetic field in the actual SNR, can be approximately taken into account by a renormalization factor $f_{\mathrm{re}}<1$, roughly $f_{\mathrm{re}}=0.15$ to 0.25 , which diminishes the nucleonic CR production efficiency calculated in the spherical model, and all effects associated with it.

We assume also that electrons are injected into the acceleration process at the shock front. Formally their injection momentum is taken to be the same as that of the protons. Since the physical processes of electron injection are still poorly known, we choose the electron injection rate such that the electron:proton ratio $K_{\text {ep }}$ (which we define as the ratio of their distribution functions $f_{\mathrm{e}}(p) / f(p)$ at all rigidities where the protons are already relativistic and the electrons have not been yet cooled radiatively) is a constant to be determined from the synchrotron observations.

The electron distribution function $f_{\mathrm{e}}(p)$ deviates only at sufficiently large momenta from the relation $f_{\mathrm{e}}(p)=K_{\mathrm{ep}} f(p)$ as a result of synchrotron losses, which are taken into account by supplementing the ordinary diffusive transport equation by a radiative loss term.

From the point of view of injection/acceleration theory, we must treat $K_{\mathrm{ep}}$, together with $B_{0}$ and $\eta$, as a theoretically until now not very precisely calculable parameter, to be quantitatively determined by comparison with the synchrotron observations (see Völk 2003; Berezhko 2005, for reviews)

The solution of the dynamic equations at each instant of time yields the CR spectrum and the spatial distributions of CRs and thermal gas. This allows the calculation of the expected fluxes of nonthermal emission produced by the accelerated CRs. 


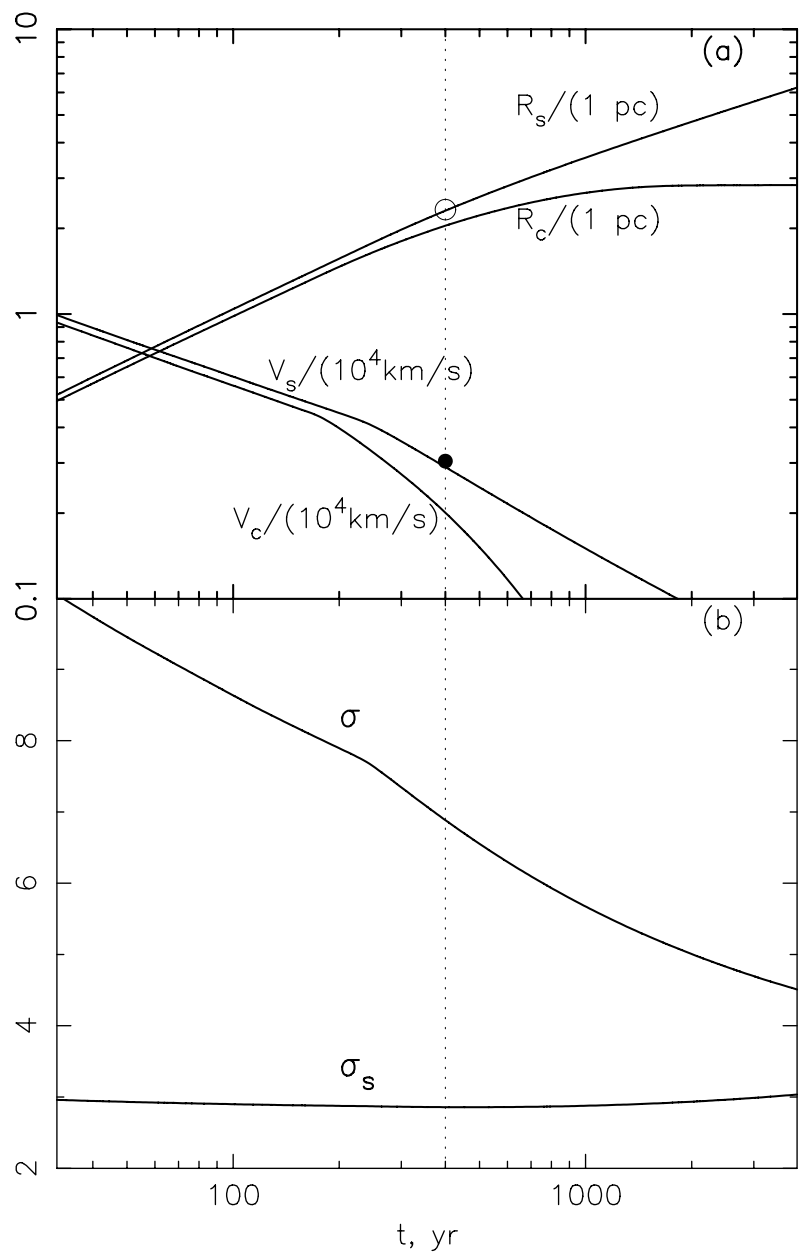

Fig. 1. a) Shock radius $R_{\mathrm{s}}$, contact discontinuity radius $R_{\mathrm{c}}$, shock speed $V_{\mathrm{s}}$, and contact discontinuity speed $V_{\mathrm{c}}$, as functions of time since explosion; b) total shock $(\sigma)$ and subshock $\left(\sigma_{\mathrm{s}}\right)$ compression ratios. The dotted vertical line marks the current epoch $t_{\mathrm{c}}$. The observed mean size and speed of the shock, as determined by radio measurements (Dickel et al. 1988), are shown as well.

\section{Results and discussion}

As a starting case we consider the typical value $E_{\text {sn }}=10^{51} \mathrm{erg}$ for the explosion energy and the conventional distance $d=4.8 \mathrm{kpc}$. The results of our calculations are presented in Figs. 1-2. The hydrogen number density $N_{\mathrm{H}}=3 \mathrm{~cm}^{-3}$, which determines the ISM density $\rho_{0}=1.4 m_{\mathrm{p}} N_{\mathrm{H}}$, was chosen to fit the size $R_{\mathrm{S}}$ and the expansion speed $V_{\mathrm{s}}$ at the present age $t_{\mathrm{c}}=400 \mathrm{yr}$. Such a value of $N_{\mathrm{H}}$ is clearly a spatial average over the actual density structure. Optical $\mathrm{H} \alpha+[\mathrm{N} \mathrm{II}]$ data show knots and filaments, also influenced by variable foreground extinction (e.g. Blair 2005, for a review). For the acceleration of the very high energy particles which give rise to the hard X-ray synchrotron and TeV $\gamma$ ray emissions such small-scale inhomogeneities, however, play essentially no role.

As shown in Fig. 1a the current evolutionary phase of Kepler's SNR corresponds to the Sedov phase. The adopted proton injection rate $\eta=1.5 \times 10^{-3}$ leads to a significant shock modification, characterized by a total shock compression ratio $\sigma \approx 6.9$ and a subshock compression ratio $\sigma_{\mathrm{s}} \approx 2.9$ (see Fig. $1 \mathrm{~b}$ ). Such a shock modification is needed to fit the observed steep radio spectrum and the smooth connection with its X-ray part (see below).

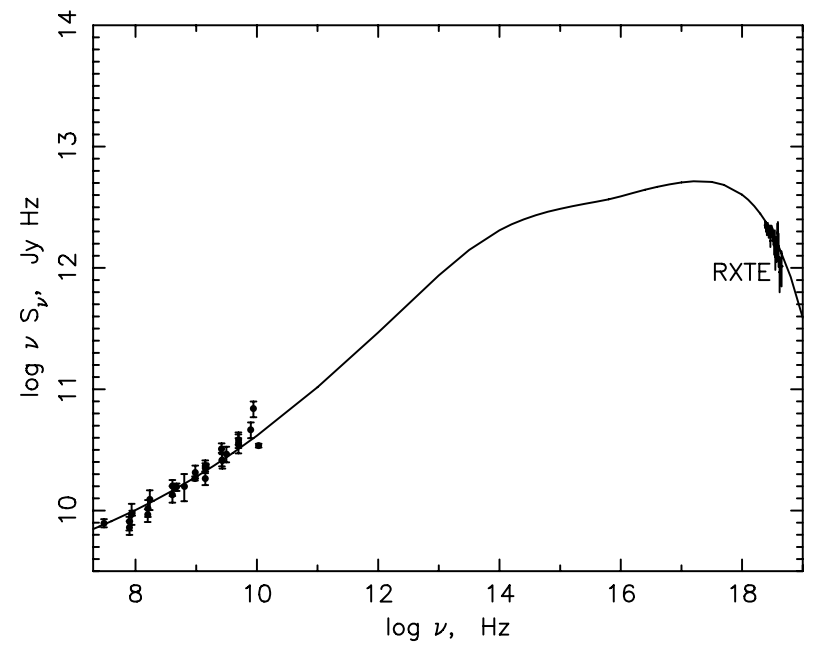

Fig. 2. Calculated energy flux of synchrotron emission as a function of frequency for the same case as in Fig. 1. The observed non-thermal X-ray Allen et al. (1999) and radio emission Reynolds \& Ellison (1992) flux values are also shown.

About $10 \%$ of the explosion energy has been transfered into $\mathrm{CR}$ energy up to now, which means that the CR energy content is $E_{\mathrm{c}}=0.1 E_{\mathrm{sn}}$.

The calculated synchrotron flux is shown in Fig. 2 together with the observed values at radio and X-ray frequencies. At radio frequencies the synchrotron spectrum $S_{v} \propto v^{-\alpha}$ has spectral index $\alpha=0.71$ (DeLaney et al. 2002). It deviates significantly from the value $\alpha=0.5$ that corresponds to an unmodified strong shock. The adopted proton injection rate $\eta=1.5 \times 10^{-3}$ gives the required shock modification. The electron-to-proton ratio $K_{\mathrm{ep}}=1.3 \times 10^{-4}$ and an interior magnetic field strength $B_{\mathrm{d}}=480 \mu \mathrm{G}$ give a good fit for the experimental data in the radio and $\mathrm{X}$-ray ranges. Note that the interior magnetic field $B_{\mathrm{d}}=480 \mu \mathrm{G}$, derived here from the fit of the overall synchrotron spectrum, is higher than the value $B_{\mathrm{d}}^{\prime}=215 \mu \mathrm{G}$, determined from the observed spatial fine structure of the synchrotron emission (Völk et al. 2005). However, if we use the thinnest X-ray radial profile observed by Chandra (Bamba et al. 2005), which has an angular width $\Delta \psi=2.1^{\prime \prime}$, we obtain $B_{\mathrm{d}}^{\prime}=340 \mu \mathrm{G}$ in rough agreement with the value derived from the fit of the overall synchrotron spectrum. Such a high interior magnetic field is the result of field amplification by the nonlinear CR backreaction on the acceleration process (Bell \& Lucek 2001; Bell 2004). It was recently established that such strong field amplification takes place in all young Galactic SNRs which have known filamentary structures in the nonthermal X-ray emission (Völk et al. 2005).

In Fig. 3 we present the gamma-ray spectrum of Kepler's SNR, expected at the current epoch. It is mainly produced by the CR proton component in hadronic collisions with background gas nuclei, leading to $\pi^{0}$-production and subsequent decay into two gamma-quanta. This so-called hadronic $\gamma$-ray component exceeds the leptonic $\gamma$-ray component due to the Inverse Compton (IC) scattering off the cosmic microwave background by more than a factor of $10^{3}$. The integral gamma-ray spectrum is expected to be very hard, $F_{\gamma} \propto \epsilon_{\gamma}^{-0.8}$, within the energy range from $1 \mathrm{GeV}$ to almost $10 \mathrm{TeV}$. At $\epsilon_{\gamma}=1 \mathrm{TeV}$ $\epsilon_{\gamma} F_{\gamma} \approx 5 \times 10^{-12} \mathrm{erg} /\left(\mathrm{cm}^{2} \mathrm{~s}\right)$ for $E_{\mathrm{sn}}=10^{51} \mathrm{erg}$. Since the SN explosion energy is not exactly known, we present in Fig. 3 also the results calculated for the three other values $E_{\mathrm{sn}} /\left(10^{51} \mathrm{erg}\right)=$ $0.5,1.5$ and 2 . We note that even at the lowest explosion energy 


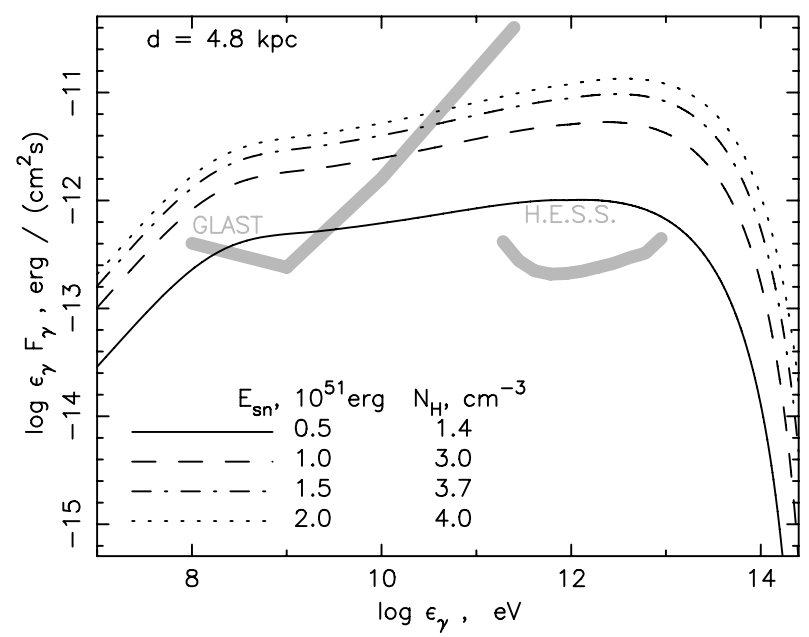

Fig. 3. Total $\left(\pi^{0}\right.$-decay + IC) integral $\gamma$-ray energy fluxes as a function of $\gamma$-ray energy for the source distance $d=4.8 \mathrm{kpc}$ and four values of the $\mathrm{SN}$ explosion energy $E_{\mathrm{sn}} /\left(10^{51} \mathrm{erg}\right)=0.5,1,1.5,2$. For comparison, the respective sensitivities for a $5 \sigma$ detection in one year with GLAST (Weekes 2003), and in $50 \mathrm{~h}$ at $20^{\circ}$ zenith angle for a photon index 2.6 (as for the Crab Nebula) with HESS (Funk 2005), are shown.

$E_{\mathrm{sn}}=0.5 \times 10^{51} \mathrm{erg}$ considered here, the expected $\gamma$-ray flux exceeds the sensitivity of the GLAST instrument at GeV energies and of the HESS instrument at $\mathrm{TeV}$ energies. At TeV-energies the expected energy flux is $\epsilon_{\gamma} F_{\gamma} \approx 10^{-12} \mathrm{erg} /\left(\mathrm{cm}^{2} \mathrm{~s}\right)$ in the case $E_{\mathrm{sn}}=0.5 \times 10^{51} \mathrm{erg}$ and an order of magnitude higher for $E_{\mathrm{sn}}=2 \times 10^{51} \mathrm{erg}$.

Qualitatively, the dependence of the expected $\gamma$-ray flux on the explosion energy can be understood if one takes into account that the three relevant parameters - distance $d$, explosion energy $E_{\mathrm{sn}}$ and ISM density $N_{\mathrm{H}}-$ are connected by the relation

$N_{\mathrm{H}} \propto E_{\mathrm{sn}} / d^{5}$

because in the Sedov phase the SNR radius $R_{\mathrm{S}} \propto d$ is determined by the relation $R_{\mathrm{S}} \propto\left(E_{\mathrm{sn}} / N_{\mathrm{H}}\right)^{1 / 5} t^{2 / 5}$. Taking into account the dependence of the $\pi^{0}$-decay $\gamma$-ray flux on the relevant parameters

$F_{\gamma} \propto E_{\mathrm{c}} N_{\mathrm{H}} / d^{2}$

together with the fact that in the Sedov phase the ratio $E_{\mathrm{c}} / E_{\mathrm{sn}}$ is a weakly dependent function of time, we have

$F_{\gamma} \propto E_{\mathrm{sn}}^{2} / d^{7}$.

For a given source distance $d$ this gives $F_{\gamma} \propto E_{\mathrm{sn}}^{2}$, in rough agreement with the numerical results presented in Fig. 3.

Note that magnetic field strengths vary very little with the explosion energy: for the interval $E_{\mathrm{sn}}=(0.5-2) \times 10^{51} \mathrm{erg}$ considered, we have $B_{\mathrm{d}}=440-500 \mu \mathrm{G}$ and $B_{\mathrm{d}}^{\prime}=346-337 \mu \mathrm{G}$, whereas the electron:proton ratio $K_{\text {ep }}=(2.8-0.7) \times 10^{-4}$ varies roughly inversely proportional to the energy $E_{\mathrm{sn}}$.

Since the source distance is not known very well, we performed our calculations for a range of distances $d=3.4-7 \mathrm{kpc}$ in a similar way as it was done above for $d=4.8 \mathrm{kpc}$. In each case we achieve the same quality of fit of the observed SNR size, its expansion speed and the overall synchrotron emission spectrum. Therefore we present in Fig. 4 only the results of the $\gamma$-ray energy fluxes expected for the SN explosion energy $E_{\mathrm{sn}}=10^{51} \mathrm{erg}$ and for four different distances from the range $d=3.4-7 \mathrm{kpc}$. It can be seen from Fig. 4 that Kepler's SNR is expected to be as bright a $\mathrm{TeV} \gamma$-ray source as the Crab Nebula if the distance

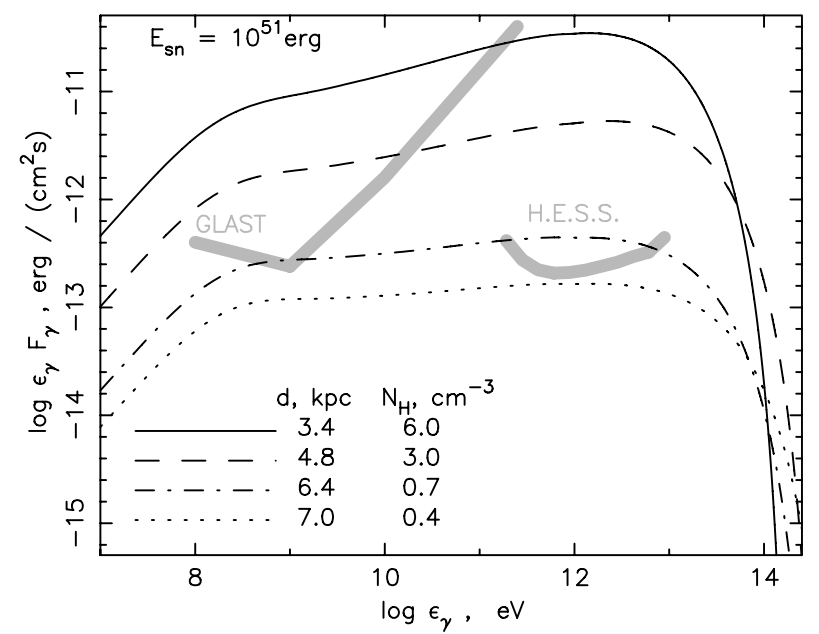

Fig. 4. Same as in Fig. 3, but different curves correspond now to different source distances $d=3.4,4.8,6.4,7 \mathrm{kpc}$ for the explosion energy $10^{51} \mathrm{erg}$.

is as small as $d=3.4 \mathrm{kpc}$. The expected $\gamma$-ray flux goes down with increasing distance and comes to the minimum observable HESS flux if the distance becomes as large as $7 \mathrm{kpc}$.

According to Eq. (2), for a fixed value of the SN explosion energy $E_{\mathrm{sn}}$ the expected $\gamma$-ray flux is

$F_{\gamma} \propto d^{-7}$

in good agreement with the results presented in Fig. 4.

We note here that according to the so-called delayed detonation model (Gamezo et al. 2005) the value $E_{\mathrm{sn}}=10^{51} \mathrm{erg}$ is even below the lower end of the expected range of type Ia SN explosion energies. From this point of view the results presented in Fig. 4 most probably give a lower limit for the expected $\gamma$-ray flux for any given distance $d$, and therefore these results convince us that Kepler's SNR is a potential high-energy $\gamma$-ray source.

We emphasize that an increase of the assumed source distance leads to an increase of the linear width $L \propto d \Delta \psi$ of the filaments observed in nonthermal X-rays. This leads in turn to a decrease of the interior magnetic field strength $B_{\mathrm{d}}^{\prime} \propto L^{-2 / 3}$, determined from the filament size. On the other hand, the linear shock size $R_{\mathrm{S}} \propto d$ and the shock speed $V_{\mathrm{s}} \propto d$ become larger for larger $d$ and this makes the CR acceleration process faster. Therefore a higher magnetic field $B_{\mathrm{d}}$ with a correspondingly more intense synchrotron cooling effect of CR electrons is required in order to provide a smooth cutoff of the synchrotron spectrum $S_{v}(v)$ consistent with the existing nonthermal X-ray measurements (see Fig. 2). As a result, at $d=7 \mathrm{kpc}$ we have $B_{\mathrm{d}}=534 \mu \mathrm{G}$, whereas $B_{\mathrm{d}}^{\prime}=295 \mu \mathrm{G}$ is already considerably lower. This means that a source distance $d \approx 7 \mathrm{kpc}$ or larger has to be considered as inappropriate if the radial X-ray profile width $\Delta \psi=2.1^{\prime \prime}$ observed by Chandra (Bamba et al. 2005) is indeed the smallest one in this source. Then Kepler's SNR has to be considered as a promising potential source of high-energy $\gamma$-rays. We note also that the electron:proton ratio $K_{\text {ep }}$ is quite insensitive to the distance $d$.

It is clear that the asymmetry of the X-ray emission is evidence that the surrounding ISM is inhomogeneous on a scale comparable with the SNR size (e.g. Bandiera 1987). Our spherically symmetrical approach is not able to take this into account. However, since we used the SNR size $R_{\mathrm{s}}$ and speed $V_{\mathrm{s}}$ as averages over the remnant, we believe that the ISM density adopted for each pair of values $d$ and $E_{\mathrm{sn}}$ also represents the ambient 
density averaged over the remnant and thus furnishes a reliable estimate for the expected total gamma-ray emission.

\section{Summary}

Our consideration of $\mathrm{CR}$ acceleration and nonthermal emission in Kepler's SNR demonstrates that a spherically symmetric nonlinear kinetic theory reproduces the SNR dynamics and the properties of its nonthermal radiation in a very satisfactory way when Kepler's SN is treated as a type Ia SN.

The $\gamma$-ray energy flux expected at $\mathrm{TeV}$ energies is $\epsilon_{\gamma} F_{\gamma} \approx$ (3-5) $\times 10^{-12} \mathrm{erg} /\left(\mathrm{cm}^{2} \mathrm{~s}\right)$ if the distance is as small as $d=$ $4.8 \mathrm{kpc}$. The flux is expected to be in a detectable range $\epsilon_{\gamma} F_{\gamma}>$ $10^{-13} \mathrm{erg} /\left(\mathrm{cm}^{2} \mathrm{~s}\right)$ at $\mathrm{TeV}$ energies if the distance does not exceed $7 \mathrm{kpc}$. If the upper limit for the source distance is indeed $d=6.4 \mathrm{kpc}$ (Reynoso \& Goss 1999) - a conclusion that is confirmed by the consistency check of the interior magnetic field values obtained by two independent methods - we conclude that Kepler's SNR is a potentially bright $\gamma$-ray source in the sky.

Acknowledgements. This work has been supported in part by the Russian Foundation for Basic Research (grant 03-02-16524) and LSS 422.2003.2. E.G.B. and L.T.K. acknowledge the hospitality of the Max-Planck-Institut für Kernphysik, where part of this work was carried out. The authors thank W. Hillebrandt and G. Pühlhofer for discussions on type Ia SN explosions and the specific properties of Kepler's SN.

\section{References}

Allen, G. E., Gotthelf, E. V., \& Petre, R. 1999, Proc. 26th ICRC, Salt Lake City, 3, 480 Baade, W. 1943, ApJ, 97, 119

Badenes, C., Borkowski, K. J., \& Bravo, E. 2005, ApJ, 624, 198

Bamba, A., Yamazaki, R., Yoshida, T., Terasawa, T., \& Koyama, K. 2005, ApJ, 621, 793 Bandiera, R. 1987, ApJ, 319, 885
Bell, A. R. 1978, MNRAS, 182, 147

Bell, A. R. 2004, MNRAS, 353, 550

Bell, A. R., \& Lucek, S. G. 2001, MNRAS, 321, 433

Berezhko, E. G. 2005, Adv. Space Res., 35, 1031

Berezhko, E. G., Elshin, V. K., \& Ksenofontov, L. T. 1996, J. Exp. Theor. Phys., 82, 1

Berezhko, E. G., Ksenofontov, L. T., \& Völk, H. J. 2002, A\&A, 395, 943

Berezhko, E. G., Ksenofontov, L. T., \& Völk, H. J. 2003a, A\&A, 412, L11

Berezhko, E. G., Pühlhofer, G., \& Völk, H. J. 2003b, A\&A, 400, 971

Berezhko, E. G., \& Völk, H. J. 1997, Astropart. Phys., 7, 183

Berezhko, E. G., \& Völk, H. J. 2004, A\&A, 419, L27

Blair, W. P. 2005, in Supernovae as Cosmological Lighthouses, ASP Conf. Ser., 342, 416

Blandford, R. D., \& Ostriker, J. P. 1978, ApJ, 221, L29

Borkowski, K. J., Blondin, J. M., \& Sarazin, C. L. 1992, ApJ, 400, 222

Cassam-Chenaï, G., Decourchelle, A., Ballet, J., et al. 2004, A\&A, 414, 545

Chevalier, R. A. 1982, ApJ, 258, 790

DeLaney, T., Koralesky, B., Rudnick, L., \& Dickel, J. R. 2002, ApJ, 580, 914

Dickel, J. R., Sault, R., Arendt, R. G., Korista, K. T., \& Matsui, Y. 1988, ApJ, 330, 254

Doggett, J. B., \& Branch, D. 1985, AJ, 90, 2303

Funk, S. 2005, in Ph.D. Thesis, Univ. of Heidelberg, Germany, 71

Gamezo, V. N., Khokhlov, A. M., \& Oran, E. S. 2005, ApJ, 623, 337

Hillebrandt, W. 2004, New A Rev., 48, 615

Hwang, U., Holt, S. S., Petre, R., Szymkowiak, A. E., \& Borkowski, K. J. 2000, BAAS, 32, 1236

Jones, E. M., Smith, B. W. \& Straka, W. C. 1981, ApJ, 249, 185

Khokhlov, A. M. 2000 [arXiv: astro-ph/0008463]

Kinugasa, K., \& Tsunemi, H. 1999, PASJ, 51, 239

Lucek, S. G., \& Bell, A. R. 2000, MNRAS, 314, 65

Malkov, M. A., \& Drury, L. O. 2001, Rep. Progress Phys., 64, 429

McKenzie, J. F., \& Völk, H. J. 1982, A\&A, 116, 191

Reinecke, M., Hillebrandt, W., \& Niemeyer, J. C. 2002, A\&A, 386, 936

Reynolds, S. P., \& Ellison, D. C. 1992, ApJ, 399, L75

Reynoso, E. M., \& Goss, W. M. 1999, AJ, 118, 926

Völk, H. J., Berezhko, E. G., \& Ksenofontov, L. T. 2003, A\&A, 409, 563

Völk, J. H., \& McKenzie, F. J. 1981, in Proc. 17th ICRC, Paris, 246

Völk, H. J. 2003, in Proc. 28th ICRC (Tsukuba), ed. T. Kajita, Y. Asaoka, A. Kawachi, Y. Matsubara, \& M. Sasaki (Tokyo, Japan: Universal Academy Press Inc.), 8, 29

Völk, H. J., Berezhko, E. G., \& Ksenofontov, L. T. 2005, A\&A, 433, 229

Völk, H. J., Berezhko, E. G., Ksenofontov, L. T., \& Rowell, G. P. 2002, A\&A, 396, 649

Weekes, T. C. 2003, in Proc. 28th ICRC (Tsukuba), ed. T. Kajita, Y. Asaoka, A. Kawachi,

Y. Matsubara, \& M. Sasaki (Tokyo, Japan: Universal Academy Press Inc.), 8, 3

Wheeler, J. C., Harkness, R. P., Khokhlov, A. M., \& Hoflich, P. 1995, Phys. Rep., 256, 211 